\title{
ABOUT A NON-STANDARD INTERPOLATION PROBLEM
}

\author{
DANIEL ALPAY AND ALAIN YGER
}

\begin{abstract}
Using algebraic methods, and motivated by the one variable case, we study a multipoint interpolation problem in the setting of several complex variables. The duality realized by the residue generator associated with an underlying Gorenstein algebra, using the Lagrange interpolation polynomial, plays a key role in the arguments.
\end{abstract}

\section{Contents}

1. Introduction

2. Zero dimensional polynomials ideals in $\mathbb{C}\left[s_{1}, \ldots, s_{n}\right]$ and duality

3. Cauchy-Weil's integral representation formula and Lagrange interpolation

4. Two non-standard interpolation problems

5. Conclusions

References

\section{INTRODUCTION}

In [3] the following multipoint interpolation problem was considered:

Problem 1.1. Given complex numbers $a_{j, k}\left(j=1, \ldots, m\right.$ and $\left.k=0, \ldots, \mu_{j}-1\right)$ and $c$, describe the set of all functions $f$ analytic in a neighborhood $\Omega$ of the points $w_{1}, \ldots, w_{m}$ and such that

$$
\sum_{j=1}^{m} \sum_{k=0}^{\mu_{j}-1} a_{j, k} f^{(k)}\left(w_{j}\right)=c
$$

Note that if $f$ solves (1.1) so does $f+p h$, where

$$
p(z)=\prod_{j=1}^{n}\left(z-w_{j}\right)^{\mu_{j}}, \quad N=\sum_{j=0}^{m} \mu_{j} .
$$

In other words one can work in the ideal $H(\Omega) /(p)$. In 3 one used a different approach and a key tool to solve the above problem was to represent any function analytic in $\Omega$ in the form

$$
f(z)=\sum_{\nu=0}^{N-1} z^{\nu} f_{\nu}(p(z)),
$$

and $f_{0}, \ldots, f_{N-1}$ are analytic in a neighborhood of the origin. This representation allows to reduce condition (1.1) to a tangential interpolation condition at the origin for the $\mathbb{C}^{N}$-valued function $F=\left[\begin{array}{lll}f_{0} & \cdots & f_{N-1}\end{array}\right]^{t}$.

Date: July 22, 2018.

2010 Mathematics Subject Classification. Primary 32A27; Secondary 13P.

Key words and phrases. Residue theory; interpolation.

The authors thank the Foster G. and Mary McGaw Professorship in Mathematical Sciences, which supported this research. 
In the present paper we study the counterpart of the previous interpolation problem in the setting of several complex variables, see Problems 4.1 and 4.2 below. We now replace the polynomial (1.2) by a zero-dimensional ideal in $\mathbb{C}[s]$ generated by $n$ polynomials $p_{1}, \ldots, p_{n}$, and characterize the elements in the corresponding quotient space in terms of a duality realized by the residue generator associated with the Gorenstein algebra $\mathbb{C}[\mathbf{s}] /(p)$, using the Lagrange interpolation polynomial (see (3.4) for the latter). This allows to define local coordinates and then translate the interpolation condition into an hyperplan condition in terms of these coordinates. Thus both in the one variable approach of [3] and in the present work one reduces condition (1.1) to a single interpolation condition.

\section{Zero DimEnSiONAL POLYNOMIALS IDEALS IN $\mathbb{C}\left[s_{1}, \ldots, s_{n}\right]$ AND DUALITY}

Notations. In the polynomial algebra $\mathbb{C}[\boldsymbol{s}]\left(\boldsymbol{s}=\left(s_{1}, \ldots, s_{n}\right)\right)$, one will denote, for any $\boldsymbol{\beta} \in \mathbb{N}^{n}$ as $\boldsymbol{s}^{\boldsymbol{\beta}}$ the monomial $s_{1}^{\beta_{1}} \ldots s_{n}^{\beta_{n}}$. Given two elements $\boldsymbol{\ell}$ and $\boldsymbol{\ell}^{\prime}$ in $\mathbb{N}^{n}$, by $\ell \prec \ell^{\prime}$, we mean $\ell_{j} \leq \ell_{j}^{\prime}$ for any $j=1, \ldots, n$. We also denote $|\ell|:=\ell_{1}+\cdots+\ell_{n}$ and $\ell !:=\ell_{1} ! \cdots \ell_{n} !$

A polynomial ideal $(\boldsymbol{p})=\left(p_{1}, \ldots, p_{M}\right)$ in $\mathbb{C}[\boldsymbol{s}]$ is said to be zero-dimensional if its zero set $\boldsymbol{p}^{-1}(0)=\left\{\boldsymbol{\zeta} \in \mathbb{C}^{n} ; p_{1}(\boldsymbol{\zeta})=\cdots=p_{M}(\boldsymbol{\zeta})=0\right\}$ is non-empty and discrete, hence finite since it is an algebraic subvariety in the affine space $\mathbb{A}_{\mathbb{C}}^{n}$. When additionally the number of polynomial generators equals the dimension, that is $M=n$, the set of generators $\left(p_{1}, \ldots, p_{n}\right)$ is said to define a discrete complete intersection in $\mathbb{C}^{n}$ (or, equivalently, the sequence $\left(p_{1}, \ldots, p_{n}\right)$ is a quasi-regular sequence in $\left.\mathbb{C}[\boldsymbol{s}]\right)$.

It is equivalent to say that $(\boldsymbol{p})=\left(p_{1}, \ldots, p_{M}\right)$ is zero-dimensional and that the $\mathbb{C}$ vector space $\mathbb{C}[\boldsymbol{s}] /(\boldsymbol{p})$ is finitely dimensional, with

$$
\operatorname{dim}_{\mathbb{C}}(\mathbb{C}[\boldsymbol{s}] /(\boldsymbol{p}))=N(\boldsymbol{p}) \leq d_{1} d_{2} \cdots d_{n}
$$

(provided $d_{2}=\operatorname{deg} p_{2} \geq d_{3}=\operatorname{deg} p_{3} \geq \cdots \geq d_{M}=\operatorname{deg} p_{M} \geq d_{1}=\operatorname{deg} p_{1}$ ); this follows from Bézout geometric theorem. In order to construct a monomial basis $\mathscr{B}_{\mathbb{C}[\boldsymbol{s}] /(\boldsymbol{p})}^{\prec \prec}=\left\{\dot{\boldsymbol{s}}^{\boldsymbol{\beta}_{k}} ; k=0, \ldots, N(\boldsymbol{p})-1\right\}$ for $\mathbb{C}[\boldsymbol{s}] /(\boldsymbol{p})$, which will be required in order to settle the results presented in this paper, one proceeds algorithmically as follows :

- decide of an order $\prec \prec$ on $\mathbb{N}^{n}$ (e.g the reverse lexicographic order, which is the most currently used) ;

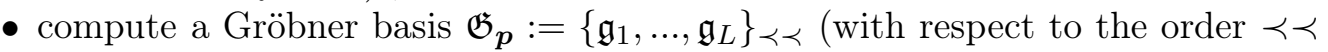
fixed from the beginning) ;

- collect all monomials that do not belong to the monomial ideal generated by the leading monomials (comparing their multi-exponents in terms of the order $\prec \prec)$ of the polynomial entries in $\mathfrak{G}_{p}$.

Example 2.1. In the particular case where $M=n$ and each $p_{j}$ is a univariate polynomial with degree $d_{j}$ in the single variable $s_{j}$, a monomial basis $\mathscr{B}_{\mathbb{C}[s] /(\boldsymbol{p})}^{d}$ is provided (thanks to the Euclidean division algorithm with respect successively to the variables $\left.s_{1}, \ldots, s_{n}\right)$ as

$$
\mathscr{B}_{\mathbb{C}[s] /(\boldsymbol{p})}^{\boldsymbol{d}}=\mathscr{B}_{\boldsymbol{p}}^{\text {euclid }}:=\left\{\dot{\boldsymbol{s}}^{\boldsymbol{\beta}} ; \boldsymbol{\beta} \in \mathbb{N}^{n} \text { with } \boldsymbol{\beta} \prec \boldsymbol{d}-\mathbf{1}\right\} \quad\left(\boldsymbol{d}:=\left(d_{1}, \ldots, d_{n}\right)\right) .
$$

Zero-dimensional ideals in $\mathbb{C}[s]$ that will be of interest for us in this paper will be generated by exactly $n$ polynomials $\left(p_{1}, \ldots, p_{n}\right)$ (defining then a quasi-regular sequence in $\mathbb{C}[\boldsymbol{s}])$. In such a case, one can find a matrix $\boldsymbol{A}=\left[a_{j, k}\right] \in \mathscr{M}_{n, n}(\mathbb{C}[\boldsymbol{s}])$ and $n$ univariate 
polynomials $q_{1}\left(s_{1}\right), \ldots, q_{n}\left(s_{n}\right)$ such that

$$
\left[\begin{array}{c}
q_{1}\left(s_{1}\right) \\
\vdots \\
q_{n}\left(s_{n}\right)
\end{array}\right]=\boldsymbol{A}(\boldsymbol{s}) \cdot\left[\begin{array}{c}
p_{1}(\boldsymbol{s}) \\
\vdots \\
p_{n}(\boldsymbol{s})
\end{array}\right]
$$

with $\max _{j, k}\left(\operatorname{deg} q_{j}, \operatorname{deg} p_{j}+\operatorname{deg} a_{j, k}\right) \leq d_{1} \cdots d_{n}$ (resp. $\left.\leq d_{1} \cdots d_{n} d_{n+1}\right)$, see [14].

Given a zero-dimensional ideal $(\boldsymbol{p})=\left(p_{1}, \ldots, p_{M}\right)$, the finitely dimensional $\mathbb{C}$-vector space $\operatorname{Hom}_{\mathbb{C}}(\mathbb{C}[\boldsymbol{s}] /(\boldsymbol{p}), \mathbb{C})$ inherits a structure of $\mathbb{C}[\boldsymbol{s}] /(\boldsymbol{p})$-module, setting

$$
\forall \dot{h} \in \mathbb{C}[\boldsymbol{s}] /(\boldsymbol{p}), \quad \forall \Phi \in \operatorname{Hom}_{\mathbb{C}}(\mathbb{C}[\boldsymbol{s}] /(\boldsymbol{p}), \mathbb{C}), \quad \dot{h} \cdot \Phi=\Phi \circ \boldsymbol{h},
$$

where $\boldsymbol{h}$ denotes the element in $\operatorname{Hom}_{\mathbb{C}}(\mathbb{C}[\boldsymbol{s}] /(\boldsymbol{p}), \mathbb{C}[\boldsymbol{s}] /(\boldsymbol{p}))$ which is induced by the multiplication by $h$.

In the particular case where $M=n$ and $\boldsymbol{p}=\left(p_{1}, \ldots, p_{n}\right)$ is a quasi-regular sequence, the $\mathbb{C}[\boldsymbol{s}] /(\boldsymbol{p})$-module $\mathbb{C}[\boldsymbol{s}] /(\boldsymbol{p}) \cdot \operatorname{Hom}_{\mathbb{C}}(\mathbb{C}[\boldsymbol{s}] /(\boldsymbol{p}), \mathbb{C})$ defined as in (2.3) is generated by the single element

$$
\dot{h} \longmapsto \operatorname{Res}\left[\begin{array}{c}
h(\boldsymbol{s}) d s_{1} \wedge \cdots \wedge d s_{n} \\
p_{1}(s), \ldots, p_{n}(s)
\end{array}\right]=\operatorname{Res}\left[\begin{array}{c}
h(\boldsymbol{s}) \operatorname{det}[\boldsymbol{A}(s)] d s_{1} \wedge \cdots \wedge d s_{n} \\
q_{1}\left(s_{1}\right), \ldots, q_{n}\left(s_{n}\right)
\end{array}\right],
$$

where the univariate polynomials $q_{j}$ and the matrix $\boldsymbol{A} \in \mathscr{M}_{n, n}(\mathbb{C}[s])$ satisfy (2.2) (independently of the choice of the $q_{j}\left(s_{j}\right)$ and $\boldsymbol{A}$ such that such the matricial identity (2.2) holds). If $\boldsymbol{d}_{q}=\left(\operatorname{deg} q_{1}, \ldots, \operatorname{deg} q_{n}\right)$ and $\mathbf{1}=(1, \ldots, 1)$, the right-hand side of (2.4) equals the coefficient $\tau_{\boldsymbol{d}_{q}-\mathbf{1}}$ of the monomial $s^{\boldsymbol{d}_{q}-\mathbf{1}}$ in the remainder $\sum_{\boldsymbol{\ell} \prec \boldsymbol{d}_{q}-\mathbf{1}} \tau_{\boldsymbol{\ell}} \boldsymbol{s}^{\boldsymbol{\ell}}$ after the successive euclidean divisions respectively by $q_{1}\left(s_{1}\right)=s_{1}^{\operatorname{deg} q_{1}}+\cdots, \ldots, q_{n}\left(s_{n}\right)=$ $s_{n}^{\operatorname{deg} q_{n}}+\cdots$ of the multivariate polynomial $h(\boldsymbol{s}) \operatorname{det}[\boldsymbol{A}(\boldsymbol{s})]$ ( $h$ being any representant of $\dot{h}$ ), see for example [12]. The following important equivalence materializes in this case algebraic duality :

$$
\forall F \in \mathbb{C}[s], \quad F \in \sum_{j=1}^{n} \mathbb{C}[s] p_{j} \Longleftrightarrow \forall \Phi \in \mathbb{C}[s], \operatorname{Res}\left[\begin{array}{c}
\Phi(s) F(s) d s_{1} \wedge \cdots \wedge d s_{n} \\
p_{1}(s), \ldots, p_{n}(s)
\end{array}\right]=0,
$$

which amounts to say that the quadratic form

$$
\boldsymbol{Q}_{\boldsymbol{p}}:(\dot{\Phi}, \dot{\Psi}) \in(\mathbb{C}[\boldsymbol{s}] /(\boldsymbol{p}))^{2} \longmapsto \operatorname{Res}\left[\begin{array}{c}
\Phi(\boldsymbol{s}) \Psi(\boldsymbol{s}) d s_{1} \wedge \cdots \wedge d s_{n} \\
p_{1}(s), \ldots, p_{n}(s)
\end{array}\right] \in \mathbb{C}
$$

is non-degenerated. The matrix of this non-degenerated quadratic form expressed in the monomial basis $\mathscr{B} \underset{\mathbb{C}[\boldsymbol{s}] /(\boldsymbol{p})}{\prec}=\left\{\dot{\boldsymbol{s}}^{\boldsymbol{\beta}_{k}} ; k=0, \ldots, N(\boldsymbol{p})-1\right\}$ for the $\mathbb{C}$-finite dimensional vector space $\mathbb{C}[\boldsymbol{s}] /(\boldsymbol{p})$ is then

$$
\begin{aligned}
\boldsymbol{Q}_{\boldsymbol{p}}\left[\mathscr{B}_{\mathbb{C}[\boldsymbol{s}] /(\boldsymbol{p})}^{\prec \prec}\right] & {\left[\operatorname{Res}\left[\begin{array}{c}
s_{\boldsymbol{\beta}_{1}}+\boldsymbol{\beta}_{k_{2}} d s_{1} \wedge \cdots \wedge d s_{n} \\
p_{1}(s), \ldots, p_{n}(s)
\end{array}\right]\right]_{0 \leq k_{1}, k_{2} \leq N(\boldsymbol{p})-1} } \\
& =\left[\operatorname{Res}\left[\begin{array}{c}
s^{\boldsymbol{\beta}_{k_{1}}+\boldsymbol{\beta}_{k_{2}}} \operatorname{det}[\boldsymbol{A}(\boldsymbol{s})] d s_{1} \wedge \cdots \wedge d s_{n} \\
q_{1}\left(s_{1}\right), \ldots, q_{n}\left(s_{n}\right)
\end{array}\right]_{0 \leq k_{1}, k_{2} \leq N(\boldsymbol{p})-1}\right.
\end{aligned}
$$

When $M=n$, one can attach to the homomorphism (2.4) a unique complex valued $(0, n)$ current $\bigwedge_{j=1}^{n} \bar{\partial}\left(1 / p_{j}\right)$ in $\mathbb{C}^{n}$ such that, whenever $U$ is an open subset of $\mathbb{C}^{n}$, 
$\bar{h} \bigwedge_{j=1}^{n}\left(\bar{\partial}\left(1 / p_{j}\right)\right)_{\mid U}=0$ as a $(0, n)$-current in $U$ for any $h \in H(U)$ which vanishes on $\boldsymbol{p}^{-1}(0)$ and moreover

$$
\forall \dot{h} \in \mathbb{C}[\boldsymbol{s}] /(\boldsymbol{p}), \quad\left\langle\bigwedge_{j=1}^{n} \bar{\partial}\left(1 / p_{j}\right), h(s) d s_{1} \wedge \cdots \wedge d s_{n}\right\rangle=\operatorname{Res}\left[\begin{array}{c}
h(s) d s_{1} \wedge \cdots \wedge d s_{n} \\
p_{1}(s), \ldots, p_{n}(s)
\end{array}\right] .
$$

Such a current can be defined in several ways. One of the most robust ones is the following (see [8], [16]) : for any $(n, 0)$-test form $\varphi(s) d s_{1} \wedge \cdots \wedge d s_{n}$ where $\varphi \in \mathscr{D}\left(\mathbb{C}^{n}, \mathbb{C}\right)$ is compactly supported sufficiently close from $\boldsymbol{p}^{-1}(0)$, the holomorphic mapping

$$
\begin{aligned}
\varpi=\left(\varpi_{1}, \ldots, \varpi_{n}\right) \in\left\{\varpi \in \mathbb{C}^{n} ; \operatorname{Re} \varpi_{j}>1 \text { for } j=1, \ldots, n\right\} \longmapsto \\
\frac{1}{(2 i \pi)^{n}} \int_{\mathbb{C}^{n}} \bar{\partial}\left(\frac{\left|p_{n}(s)\right|^{2 \varpi_{n}}}{p_{n}(\boldsymbol{s})}\right) \wedge \cdots \wedge \bar{\partial}\left(\frac{\left|p_{1}(\boldsymbol{s})\right|^{2 \varpi_{1}}}{p_{1}(\boldsymbol{s})}\right) \wedge \varphi(\boldsymbol{s}) d s_{1} \wedge \cdots \wedge d s_{n}
\end{aligned}
$$

extends as an holomorphic function in $\left\{\varpi \in \mathbb{C}^{n} ; \operatorname{Re} \varpi_{j}>-\eta\right\}$ for some $\eta>0$, which value at $\varpi=0$ equals precisely $\left\langle\bigwedge_{j=1}^{n} \bar{\partial}\left(1 / p_{j}\right), \varphi d s_{1} \wedge \cdots \wedge d s_{n}\right\rangle$.

Example 2.2. If $p_{1}, \ldots, p_{n}$ are $n$ univariate monic polynomials in the respective variables $s_{1}, \ldots, s_{n}$ with

$$
p_{j}(s)=p_{j}\left(s_{j}\right)=\prod_{\kappa_{j}=1}^{m_{j}}\left(s_{j}-\xi_{j, \kappa_{j}}\right)^{\nu_{j, \kappa_{j}}}, j=1, \ldots, n,
$$

one has

$$
\begin{aligned}
\left\langle\bigwedge_{j=1}^{n} \bar{\partial}\left(1 / p_{j}\right), \varphi(s) d s_{1} \wedge \cdots \wedge d s_{n}\right\rangle & =\sum_{\kappa_{1}=1}^{m_{1}} \cdots \sum_{\kappa_{n}=1}^{m_{n}}\left(\prod_{j=1}^{n} \frac{1}{\left(\nu_{j, \kappa_{j}}-1\right) !}\right) \\
& \left(\frac{\partial^{\nu_{1, \kappa_{1}}-1}}{\partial s_{1}^{\nu_{1, \kappa_{1}}-1}} \circ \cdots \circ \frac{\partial^{\nu_{n, \kappa_{n}}-1}}{\partial s_{n}^{\nu_{n, \kappa_{n}}-1}}\right)\left[\varphi(s) \prod_{j=1}^{n} \frac{\left(s_{j}-\xi_{j, \kappa_{j}}\right)^{\nu_{j, \kappa_{j}}}}{p_{j}\left(s_{j}\right)}\right]\left(\xi_{1, \kappa_{1}}, \ldots, \xi_{n, \kappa_{n}}\right) .
\end{aligned}
$$

The analytic pendant of the realization of algebraic duality (2.5) is then :

$$
\begin{aligned}
& \forall U \text { open subset of } \mathbb{C}^{n}, \quad \forall f \in H(U), \quad f \in\left(\sum_{j=1}^{n} H(U) p_{j}\right)_{\text {loc }} \\
& \Longleftrightarrow \forall \varphi \in \mathscr{D}(U, \mathbb{C}),\left\langle\bigwedge_{j=1}^{n} \bar{\partial}\left(1 / p_{j}\right), f(\boldsymbol{s}) \varphi(\boldsymbol{s}) d s_{1} \wedge \cdots \wedge d s_{n}\right\rangle=0 .
\end{aligned}
$$

In order to describe more precisely the current $\bigwedge_{j=1}^{n} \bar{\partial}\left(1 / p_{j}\right)$ when $\left(p_{1}, \ldots, p_{n}\right)$ is a quasi-regular sequence in $\mathbb{C}[s]$ (as we did in Example 2.2 in the particular case where each $p_{j}$ is univariate in the single variable $s_{j}$, see (2.9)), we need to recall how each of the distinct points $w_{j}, j=1, \ldots, m$, of the set $\boldsymbol{p}^{-1}(0)$ is equipped with a multiplicity $\nu_{w_{j}}(\boldsymbol{p}) \in \mathbb{N}^{*}$. Given $w_{j} \in \boldsymbol{p}^{-1}(0)$, such an integer $\nu_{w_{j}}(\boldsymbol{p})$ can be defined in two ways :

- "algebraically", as the dimension of the $\mathbb{C}$-vector space $\mathscr{O}_{\mathbb{C}^{n}, w_{j}} /(\boldsymbol{p})_{w_{j}}$, where $(\boldsymbol{p})_{w_{j}}$ denotes the ideal generated by the germs at $w_{j}$ of the polynomials $p_{1}, \ldots, p_{n}$ in the local regular ring $\mathscr{O}_{\mathbb{C}^{n}, w_{j}}$ of germs of holomorphic functions about the point $w_{j}$;

- "dynamically", as the number of points in the fiber $\boldsymbol{p}^{-1}(\boldsymbol{\eta})$ which remain close to $w_{j}$ when $\boldsymbol{\eta} \in\left(\mathbb{C}^{*}\right)^{n}$ tends to $\mathbf{0}$ in $\mathbb{C}^{n}$ and is taken as a non-critical value for the polynomial map $\boldsymbol{p}$. 
If one uses the first definition, it is easy to see that

$$
N(\boldsymbol{p})=\operatorname{dim}_{\mathbb{C}}(\mathbb{C}[\boldsymbol{s}] /(\boldsymbol{p}))=\sum_{j=1}^{m} \nu_{w_{j}}(\boldsymbol{p}) .
$$

It follows from (2.10), together with the fact that the Noether exponent of the ideal $(\boldsymbol{p})_{w_{j}}$ in $\mathscr{O}_{\mathbb{C}^{n}, w_{j}}$ is bounded from above by $n \nu_{j}(\boldsymbol{p})$ (see [15, 17]), that the order of the current $\bigwedge_{j=1}^{n} \bar{\partial}\left(1 / f_{j}\right)$ about the point $w_{j}$ is at most $n \nu_{w_{j}}(\boldsymbol{p})-1$. Therefore there exists a collection of differential operators $\mathscr{Q}_{w_{1}}(\partial / \partial \boldsymbol{s}), \ldots, \mathscr{Q}_{w_{m}}(\partial / \partial \boldsymbol{s}) \in \mathbb{C}[\partial / \partial \boldsymbol{s}]$ (where $\left.\partial / \partial \boldsymbol{s}=\left(\partial / \partial s_{1}, \ldots, \partial / \partial s_{n}\right)\right)$ such that $\operatorname{deg}_{\partial / \partial \boldsymbol{s}} \mathscr{Q}_{w_{j}} \leq n \nu_{w_{j}}(\boldsymbol{p})-1$ for $j=1, \ldots, m$ and

$$
\begin{aligned}
&\left\langle\bigwedge_{j=1}^{n} \bar{\partial}\left(1 / p_{j}\right), \varphi(s) d s_{1} \wedge \cdots \wedge d s_{n}\right\rangle=\sum_{j=1}^{n} \mathscr{Q}_{w_{j}}(\partial / \partial \boldsymbol{s})[\varphi]\left(w_{j}\right) \\
& \forall \varphi \in \mathscr{D}\left(\mathbb{C}^{n}, \mathbb{C}\right) .
\end{aligned}
$$

Example 2.3. If $p_{1}, \ldots, p_{n}$ are monic univariate polynomials respectively in the variables $s_{1}, \ldots, s_{n}$ as in Example 2.2 (more precisely of the form (2.8)), the multiplicity $\nu_{w}(\boldsymbol{p})$ at the point $w=\left(\xi_{1, \kappa_{1}}, \ldots, \xi_{n, \kappa_{n}}\right)$ equals $\prod_{j=1}^{n} \nu_{j, \kappa_{j}}$ and the order of the differential operator $\mathscr{Q}_{w}(\partial / \partial \boldsymbol{s})$ attached to such $w \in \boldsymbol{p}^{-1}(0)$ as in (2.9) equals in this case $\prod_{j=1}^{n}\left(\nu_{j, \kappa_{j}}-1\right)$, which happens to be strictly less than $n \nu_{w}(\boldsymbol{p})-1$ which should stand for the estimate of $\operatorname{deg} \mathscr{Q}_{w}$ in the general case. In the general case estimates $\operatorname{deg} \mathscr{Q}_{w_{j}} \leq n \nu_{w_{j}}(\boldsymbol{p})-1(j=1, \ldots, m)$ cannot in fact be sharpened.

When $U$ is an open subset of $\mathbb{C}^{n}, f \in H(U)$ and $\varphi \in \mathscr{D}(U, \mathbb{C})$, it follows from the Leibniz rule, together with the symetry of the left-hand side expression in $(\varphi, f)$ from the computational point of view, that one can write

$$
\begin{aligned}
& \left\langle\bigwedge_{j=1}^{n} \bar{\partial}\left(1 / p_{j}\right), f(\boldsymbol{s}) \varphi(\boldsymbol{s}) d s_{1} \wedge \cdots \wedge d s_{n}\right\rangle \\
& =\sum_{\left\{j \in\{1, \ldots, m\} ; w_{j} \in U\right\}} \sum_{\ell \in A_{w_{j}}} \frac{\partial^{|\ell|}}{\partial \boldsymbol{s}^{\ell}}[\varphi]\left(w_{j}\right) \mathscr{Q}_{w_{j}, \ell}(\partial / \partial \boldsymbol{s})[f]\left(w_{j}\right) \\
& =\sum_{\left\{j \in\{1, \ldots, m\} ; w_{j} \in U\right\}} \sum_{\ell \in A_{w_{j}}} \frac{\partial^{|\ell|}}{\partial \boldsymbol{s}^{\ell}}[f]\left(w_{j}\right) \mathscr{Q}_{w_{j}, \ell}(\partial / \partial \boldsymbol{s})[\varphi]\left(w_{j}\right),
\end{aligned}
$$

where $A_{w_{1}}, \ldots, A_{w_{n}}$ are finite subsets of $\mathbb{N}^{n}$ which are such that

$$
\ell \in A_{w_{j}} \Longrightarrow|\ell| \leq n \nu_{w_{j}}(\boldsymbol{p})-1 \quad \forall j=1, \ldots, m
$$

and each $\mathscr{Q}_{w_{j}, \ell}\left(j=1, \ldots, m, \ell \in A_{w_{j}}\right)$ is a polynomial in $\partial / \partial s$ with total degree at most $n \nu_{w_{j}}(\boldsymbol{p})-1-|\boldsymbol{\ell}|$ with support in $A_{w_{j}}$. Note that the $A_{w_{j}}$ and the $\mathscr{Q}_{w_{j}, \ell}$ $\left(j=1, \ldots, m, \ell \in A_{w_{j}}\right)$ depend only on the differential operators $\mathscr{Q}_{w_{j}}(\partial / \partial \boldsymbol{s})$ involved in (2.11), hence only on the given polynomial quasi-regular sequence $\boldsymbol{p}=\left(p_{1}, \ldots, p_{n}\right)$.

Definition 2.4. The list of differential operators with complex coefficients (and assigned evaluations)

$$
\operatorname{Noeth}_{\boldsymbol{p}}[(\boldsymbol{p})]:=\left\{\mathscr{Q}_{w_{j}, \ell}(\partial / \partial \boldsymbol{s})_{\mid w_{j}} ; j=1, \ldots, m, \boldsymbol{\ell} \in A_{w_{j}}\right\}
$$

will be called the standard list of assigned Notherian differential operators for the ideal ( $\boldsymbol{p})$ when considered as generated by the quasi-regular sequence $\boldsymbol{p}=\left(p_{1}, \ldots, p_{n}\right)$. 
Thanks to Definition 2.4, one can reformulate (2.10) as

$$
\begin{aligned}
\forall U \text { open subset of } \mathbb{C}^{n}, \quad \forall f \in H(U), \quad f \in\left(\sum_{j=1}^{n} H(U) p_{j}\right)_{\text {loc }} \\
\Longleftrightarrow \forall w_{j} \in U, \forall \boldsymbol{\ell} \in A_{w_{j}}, \mathscr{Q}_{w_{j}, \ell}(\partial / \partial \boldsymbol{s})[f]\left(w_{j}\right)=0 .
\end{aligned}
$$

Example 2.5. When the polynomials $p_{j}$ are monic univariate polynomials respectively in the variables $s_{1}, \ldots, s_{n}$ as (2.8), one has

$$
\begin{aligned}
& \operatorname{Noeth}_{\left(p_{1}\left(s_{1}\right), \ldots, p_{n}\left(s_{n}\right)\right)}\left[\left(p_{1}\left(s_{1}\right), \ldots, p_{n}\left(s_{n}\right)\right)\right]= \\
& \left\{{\frac{\partial^{|\ell|}}{\partial s^{\ell}}}_{\mid\left(\xi_{\left.1, \kappa_{1}, \ldots, \xi_{n, \kappa_{n}}\right)}\right.} ; 1 \leq \kappa_{j} \leq m_{j} \text { for } j=1, \ldots, m, \ell \prec\left(\nu_{1, \kappa_{1}}, \ldots, \nu_{n, \kappa_{n}}\right)-\mathbf{1}\right\}
\end{aligned}
$$

\section{Cauchy-Weil's integral Representation formula and Lagrange INTERPOLATION}

Cauchy-Weil's integral representation formula (originally introduced in [19]) plays a major role in this paper. Let us briefly recall it in the particular simple case where it happens to be the most useful (one refers for example to [2, 8, 13, 10, 18] for a more detailed as well as a presentation in its generality in the analytic or algebraic context). Let $f_{1}, \ldots, f_{n}$ be $n$ holomorphic functions in a bounded open set $U \subset \mathbb{C}^{n}$ (possibly not connected) and continuous up to $\partial U$, with no common zero on $\partial U$, such that additionally there exists a matrix $\boldsymbol{B}_{\boldsymbol{f}} \in \mathscr{M}_{n, n}(H(U \times U) \cap C(\bar{U} \times U))$ with

$$
\forall j \in\{1, \ldots, n\}, \quad \forall \boldsymbol{s}, \boldsymbol{z} \in U, \quad f_{j}(\boldsymbol{s})-f_{j}(\boldsymbol{z})=\sum_{\ell=1}^{n} b_{j, \ell}(\boldsymbol{s}, \boldsymbol{z})\left(s_{j}-z_{j}\right) .
$$

Such a matrix $\boldsymbol{B}_{\boldsymbol{f}}$ (which is definitevely non unique as soon as $n>1$ ) is called an Hefer matrix or a Bézoutian matrix when the $f_{j}$ happen to be (as it will be the case in this paper) polynomial functions. The set $V(\boldsymbol{f}):=\{w \in \bar{U} ; \boldsymbol{f}(w)=0\}$ is necessarily finite since $\boldsymbol{f}$ does not vanish on $\partial U$. For almost all $\varepsilon \in\left(\mathbb{R}^{+}\right)^{n}$ such that $\|\varepsilon\|$ is small enough, the so-called Weil analytic polyhedron

$$
\Delta_{\varepsilon}:=\left\{s \in U ;\left|f_{j}(s)\right|<\varepsilon_{j}, j=1, \ldots, n\right\}
$$

is relatively compact in $U$ and, provided $\varepsilon$ is not a critical value of the smooth map $s \in U \mapsto\left(\left|f_{1}(s)\right|^{2}, \ldots,\left|f_{n}(s)\right|^{2}\right)$ (the set of such critical values being negligible in $\left(\mathbb{R}^{+}\right)^{n}$ according to A. Sard's lemma), is such its Shilov boundary

$$
\Gamma_{\text {Shilov }}\left(\overline{\Delta_{\varepsilon}}\right):=\left\{s \in \overline{\Delta_{\varepsilon}} ;\left|f_{j}(s)\right|=\varepsilon_{j}, j=1, \ldots, n\right\}
$$

is a real analytic $n$-dimensional manifold which will be oriented as follows : the $n$ differential form $\bigwedge_{j=1}^{n} d\left[\arg \left(f_{j}(\boldsymbol{s})\right)\right]$ on will be a $n$-volume form on it. We denote then as $\Gamma_{\text {Shilov }}^{+}\left(\overline{\Delta_{\varepsilon}}\right)$ the corresponding real-analytic $n$-cycle. Then any holomorphic function $f \in H(U) \cap C(\bar{U})$ can be represented in $\Delta_{\varepsilon}$ (whenever this Weil polyhedron is connected or not) as

$$
\begin{aligned}
& \forall \boldsymbol{z} \in U, \quad f(\boldsymbol{z})= \\
& \quad \frac{1}{(2 i \pi)^{n}} \int_{\Gamma_{\text {Shilov }}^{+}\left(\overline{\Delta_{\varepsilon}}\right)} f(\boldsymbol{s}) \operatorname{det}\left[\boldsymbol{B}_{\boldsymbol{f}}(\boldsymbol{s}, \boldsymbol{z})\right] \frac{d s_{1} \wedge \cdots \wedge d s_{n}}{\left(f_{1}(\boldsymbol{s})-f_{1}(\boldsymbol{z})\right) \cdots\left(f_{n}(\boldsymbol{s})-f_{n}(\boldsymbol{z})\right)} .
\end{aligned}
$$

In this section, one considers a zero-dimensional polynomial ideal generated by a quasi-regular sequence $\boldsymbol{p}=\left(p_{1}, \ldots, p_{n}\right)$, which means that $\boldsymbol{p}^{-1}(0)$ is a non-empty 
finite set $\left\{w_{1}, \ldots, w_{n}\right\}$ in $\mathbb{C}^{n}$ (supposed distinct, each of them been equipped with a multiplicity $\nu_{w_{j}}(\boldsymbol{p})$ such that $\left.\sum_{j=1}^{m} \nu_{w_{j}}(\boldsymbol{p})=\operatorname{dim}_{\mathbb{C}}(\mathbb{C}[\boldsymbol{s}] /(\boldsymbol{p}))=N(\boldsymbol{p})\right)$. In all this section, let us also suppose that a monomial basis

$$
\mathscr{B}_{\mathbb{C}[\boldsymbol{s}] /(\boldsymbol{p})}^{\prec}=\left\{\dot{\boldsymbol{s}}^{\boldsymbol{\beta}_{k}} ; k=0, \ldots, N(\boldsymbol{p})-1\right\}
$$

for $\mathbb{C}[\boldsymbol{s}] /(\boldsymbol{p})$ has been obtained thanks to the search for a Gröbner basis for $(\boldsymbol{p})$ with respect to the prescribed ordering $\prec \prec$ on monomials in $\mathbb{C}[s]$ (as recalled in section 2).

Proposition 3.1. Let $\boldsymbol{p}=\left(p_{1}, \ldots, p_{n}\right)$ and $\underset{B}{\mathbb{C}[\boldsymbol{s}] /(\boldsymbol{p})}$ as above. Let $U$ be an open subset of $\mathbb{C}^{n}$ which contains $\boldsymbol{p}^{-1}(0)$ and $f \in H(U)$. There is a unique system of coordinates $\left(\alpha_{0}[f], \ldots, \alpha_{N(\boldsymbol{p})-1}[f]\right) \in \mathbb{C}^{n}$ such that the holomorphic function

$$
\boldsymbol{s} \in U \longmapsto f(\boldsymbol{s})-\sum_{k=0}^{N(\boldsymbol{p})-1} \alpha_{k}[f] \boldsymbol{s}^{\boldsymbol{\beta}_{k}}
$$

belongs the ideal $\left(\sum_{j=1}^{n} H(U) p_{j}\right)_{\mathrm{loc}}$.

Proof. The proof of the unicity clause goes as follows : if a polynomial function which restriction to $U$ is $s \in U \mapsto \sum_{k=0}^{N(\boldsymbol{p})-1}\left(\alpha_{k}-\tilde{\alpha}_{k}\right) \boldsymbol{s}^{\beta_{k}}$ belongs to $\left(\sum_{j=1}^{n} H(U) p_{j}\right)_{\text {loc }}$, it implies since $\boldsymbol{p}^{-1}(0) \subset U$ that the polynomial $\sum_{k=0}^{N(\boldsymbol{p})-1}\left(\alpha_{k}-\tilde{\alpha}_{k}\right) \boldsymbol{s}^{\boldsymbol{\beta}_{k}}$ belongs to $\mathbb{C}[s] p_{1}+\cdots+\mathbb{C}[s] p_{n}$, that is $\alpha_{k}=\tilde{\alpha}_{k}$ for $k=0, \ldots, N(\boldsymbol{p})-1$ since the collection $\left\{\dot{\boldsymbol{\beta}}^{k} ; k=0, \ldots, N(\boldsymbol{p})-1\right\}$ is a basis of the quotient $\mathbb{C}$-vector space $\mathbb{C}[\boldsymbol{s}] /(\boldsymbol{p})$.

As for the existence, one proceeds as follows. Let $\boldsymbol{B}_{\boldsymbol{p}} \in \mathscr{M}_{n, n}(\mathbb{C}[\boldsymbol{s}, \boldsymbol{z}])$ be any Bézoutian matrix of polynomials in $2 n$ variables $(\boldsymbol{s}, \boldsymbol{z})=\left(s_{1}, \ldots, s_{n}, z_{1}, \ldots, z_{n}\right)$ such that the following polynomial identities hold in $\mathbb{C}[\boldsymbol{s}, \boldsymbol{z}]$ :

$$
p_{j}(\boldsymbol{s})-p_{j}(\boldsymbol{z})=\sum_{\ell=1}^{n} \boldsymbol{b}_{j, \ell}(\boldsymbol{s}, \boldsymbol{z})\left(s_{\ell}-z_{\ell}\right), \quad j=1, \ldots, n .
$$

Such a matrix $\boldsymbol{B}_{\boldsymbol{p}}$ always exists : one can for example either invoke the so-called Fundamental Theorem of Analysis and take

$$
\boldsymbol{b}_{\boldsymbol{p}, j, \ell}(\boldsymbol{s}, \boldsymbol{z}):=\int_{0}^{1}\left(\frac{\partial}{\partial z_{\ell}}\right)\left[p_{j}(\boldsymbol{z}+t(\boldsymbol{s}-\boldsymbol{z}))\right] d t \quad \forall j, \ell \in\{1, \ldots, n\}
$$

or better proceed iteratively as follows for each $j=1, \ldots, n$ :

$$
\begin{aligned}
p_{j}(\boldsymbol{s})-p_{j}(\boldsymbol{z})=\frac{p_{j}\left(s_{1}, s_{2}, \ldots, s_{n}\right)-p_{j}\left(z_{1}, s_{2}, \ldots, s_{n}\right)}{s_{1}-z_{1}}\left(s_{1}-z_{1}\right) \\
+\frac{p_{j}\left(z_{1}, s_{2}, s_{3}, \ldots, s_{n}\right)-p_{j}\left(z_{1}, z_{2}, s_{3}, \ldots, s_{n}\right)}{s_{2}-z_{2}}\left(s_{2}-z_{2}\right)+\cdots
\end{aligned}
$$

in order to keep track of the smallest subring $\mathbb{A} \subset \mathbb{C}$ (for example $\mathbb{A}=\mathbb{Z}$ or $\mathbb{A}=\mathbb{Q}$ ) that contains all coefficients of $\boldsymbol{p}$ (if all $p_{j}$ lie in $\mathbb{A}[\boldsymbol{s}]$, so do then all entries of such $\boldsymbol{B}_{\boldsymbol{p}}$ ). Consider then a Weil polyhedron $\Delta \subset \subset U$ subordonned to $\left(p_{1}, \ldots, p_{n}\right)$ in the open set $U$. Cauchy-Weil's integral representation formula (3.1), together with the fact that the rational function $\zeta \mapsto 1 /(1-\zeta)$ can be expanded normally on any compact of the 
unit disk $D(0,1)$ as $\sum_{\ell \geq 0} \zeta^{\ell}$, imply

$$
\begin{aligned}
\forall z \in & \Delta, \quad f(\boldsymbol{z})=\frac{1}{(2 i \pi)^{n}} \int_{\Gamma_{\text {Shilov }}^{+}(\bar{\Delta})} f(\boldsymbol{s}) \frac{\operatorname{det}\left[\boldsymbol{B}_{\boldsymbol{p}}(\boldsymbol{s}, \boldsymbol{z})\right] d s_{1} \wedge \cdots \wedge d s_{n}}{\prod_{j=1}^{n}\left(p_{j}(\boldsymbol{s})-p_{j}(\boldsymbol{z})\right)} \\
= & \frac{1}{(2 i \pi)^{n}} \int_{\Gamma_{\text {Shilov }}^{+}(\bar{\Delta})} f(\boldsymbol{s}) \frac{\operatorname{det}\left[\boldsymbol{B}_{\boldsymbol{p}}(\boldsymbol{s}, \boldsymbol{z})\right]}{\prod_{j=1}^{n} p_{j}(\boldsymbol{s})}\left(\prod_{j=1}^{n} \frac{1}{\left.1-\frac{p_{j}(\boldsymbol{z})}{p_{j}(\boldsymbol{s})}\right) d s_{1} \wedge \cdots \wedge d s_{n}}\right. \\
= & \frac{1}{(2 i \pi)^{n}} \int_{\Gamma_{\text {Shilov }}^{+}(\bar{\Delta})} f(\boldsymbol{s}) \frac{\operatorname{det}\left[\boldsymbol{B}_{\boldsymbol{p}}(\boldsymbol{s}, \boldsymbol{z})\right] d s_{1} \wedge \cdots \wedge d s_{n}}{\prod_{j=1}^{n} p_{j}(\boldsymbol{s})} \\
& +\sum_{\boldsymbol{\ell} \in \mathbb{N}^{n} \backslash \mathbf{0}}\left(\frac{1}{(2 i \pi)^{n}} \int_{\Gamma_{\text {Shilov }}^{+}(\bar{\Delta})} f(\boldsymbol{s}) \frac{\operatorname{det}\left[\boldsymbol{B}_{\boldsymbol{p}}(\boldsymbol{s}, \boldsymbol{z})\right] d s_{1} \wedge \cdots \wedge d s_{n}}{\prod_{j=1}^{n} p_{j}^{\ell_{j}+1}(\boldsymbol{s})}\right) \boldsymbol{p}^{\ell}(\boldsymbol{z}) \\
= & \frac{1}{(2 i \pi)^{n}} \int_{\Gamma_{\text {Shilov }}^{+}(\bar{\Delta})} f(\boldsymbol{s}) \frac{\operatorname{det}\left[\boldsymbol{B}_{\boldsymbol{p}}(\boldsymbol{s}, \boldsymbol{z})\right] d s_{1} \wedge \cdots \wedge d s_{n}}{\prod_{j=1}^{n} p_{j}(\boldsymbol{s})}+\sum_{j=1}^{n} p_{j}(\boldsymbol{z}) g_{\Delta, j}(\boldsymbol{z}),
\end{aligned}
$$

where $g_{\Delta, j} \in H(\Delta)$ for any $j=1, \ldots, n$. If one takes as $\left(\alpha_{0}[f], \ldots, \alpha_{N(\boldsymbol{p})-1}[f]\right)$ the vector of coordinates (in the basis $\mathscr{B} \underset{\mathbb{C}[\boldsymbol{z}] /(\boldsymbol{p}(\boldsymbol{z}))}{\prec \prec}$ ) of the class in $\mathbb{C}[\boldsymbol{z}] /(\boldsymbol{p}(\boldsymbol{z}))$ of the polynomial

$$
\frac{1}{(2 i \pi)^{n}} \int_{\Gamma_{\text {Shilov }}^{+}(\bar{\Delta})} f(\boldsymbol{s}) \frac{\operatorname{det}\left[\boldsymbol{B}_{\boldsymbol{p}}(\boldsymbol{s}, \boldsymbol{z})\right] d s_{1} \wedge \cdots \wedge d s_{n}}{\prod_{j=1}^{n} p_{j}(\boldsymbol{s})} \in \mathbb{C}[\boldsymbol{z}],
$$

one gets the required result.

Remark 3.2. The polynomial (considered here in $\mathbb{C}[\boldsymbol{z}]$ )

$$
\operatorname{Lag}[f]:=\frac{1}{(2 i \pi)^{n}} \int_{\Gamma_{\text {Shilov }}^{+}(\bar{\Delta})} f(\boldsymbol{s}) \frac{\operatorname{det}\left[\boldsymbol{B}_{\boldsymbol{p}}(\boldsymbol{s}, \boldsymbol{z})\right] d s_{1} \wedge \cdots \wedge d s_{n}}{\prod_{j=1}^{n} p_{j}(\boldsymbol{s})}
$$

depends only on the list of germs $\left[f_{w_{1}}, \ldots, f_{w_{n}}\right]$ of germs of $f$ respectively about each of the distinct points $w_{j}$ in $\boldsymbol{p}^{-1}(0)$. It then can be considered as a Lagrange interpolator of such list of germs, hence the terminology used here to denote it. More precisely let $\varphi \in \mathscr{D}(U,[0,1])$ be any test-function which equals identically 1 about each point $w_{j}$ for $j=1, \ldots, m$. It is worth to point out that $\operatorname{Lag}[f]$ expresses alternatively (independently of the choice of the Weil polyhedron $\Delta$ ) as

$$
\begin{aligned}
& \operatorname{Lag}[f]=\left\langle\left(\bigwedge_{j=1}^{n} \bar{\partial}\left(1 / f_{j}\right)\right)(\boldsymbol{s}), f(\boldsymbol{s}) \operatorname{det}\left[\boldsymbol{B}_{\boldsymbol{p}}(\boldsymbol{s}, \boldsymbol{z})\right] \varphi(\boldsymbol{s}) d s_{1} \wedge \cdots \wedge d s_{n}\right\rangle \\
& =\sum_{j=1}^{m} \mathscr{Q}_{w_{j}}(\partial / \partial \boldsymbol{s})\left[f(\boldsymbol{s}) \operatorname{det}\left[\boldsymbol{B}_{\boldsymbol{p}}(\boldsymbol{s}, \boldsymbol{z})\right]\right]\left(w_{j}\right) \\
& =\sum_{j=1}^{m} \sum_{\ell \in A_{w_{j}}} \frac{\partial^{|\ell|}}{\partial \boldsymbol{s}^{\ell}}[f]\left(w_{j}\right) \mathscr{Q}_{w_{j}, \ell}(\partial / \partial \boldsymbol{s})\left[\operatorname{det}\left[\boldsymbol{B}_{\boldsymbol{p}}(\boldsymbol{s}, \boldsymbol{z})\right]\right]\left(w_{j}\right) \\
& =\sum_{j=1}^{m} \sum_{\ell \in A_{w_{j}}} \frac{\partial^{|\boldsymbol{\ell}|}}{\partial \boldsymbol{s}^{\ell}}\left[\operatorname{det}\left[\boldsymbol{B}_{\boldsymbol{p}}(\boldsymbol{s}, \boldsymbol{z})\right]\right]\left(w_{j}\right) \mathscr{Q}_{w_{j}, \ell}(\partial / \partial \boldsymbol{s})[f]\left(w_{j}\right),
\end{aligned}
$$

where the differential operators with complex coefficients $\mathscr{Q}_{w_{j}}(\partial / \partial s)$ for $j=1, \ldots, m$ (respectively the finite sets $A_{w_{j}} \in \mathbb{N}^{n}$ together with differential operators $\mathscr{Q}_{w_{j}, \ell}$ for $j=1, \ldots, m$ and $\ell \in A_{w_{j}}$ ) are those introduced in (2.11) (respectively in (2.12)). We refer here the reader for example to [8] or to the more up-to-date survey [18]. 
Proposition 3.3. Let $\boldsymbol{p}$ and $\mathscr{B} \underset{\mathbb{C}[s] /(\boldsymbol{p})}{\prec \prec}$ as above. Let $\left[h_{w_{1}}, \ldots, h_{w_{m}}\right]$ be a list of $m$ germs of holomorphic functions, each respectively about the zero $w_{j}$ of $\boldsymbol{p}$. There is a unique system of coordinates $\left(\alpha_{0}\left(\left[\boldsymbol{h}_{\boldsymbol{w}}\right]\right), \ldots, \alpha_{N(\boldsymbol{p})-1}\left(\left[\boldsymbol{h}_{\boldsymbol{w}}\right]\right)\right) \in \mathbb{C}^{n}$ such that for each $j=1, \ldots, m$, one has, as elements in the local ring $\mathscr{O}_{\mathbb{C}^{n}, w_{j}}$,

$$
h_{w_{j}}-\left(\boldsymbol{s} \mapsto \sum_{k=0}^{N(\boldsymbol{p})-1} \alpha\left(\left[\boldsymbol{h}_{\boldsymbol{w}}\right]\right) \boldsymbol{s}^{\boldsymbol{\beta}_{k}}\right)_{w_{j}} \in \mathscr{O}_{\mathbb{C}^{n}, w_{j}} p_{1, w_{j}}+\cdots+\mathscr{O}_{\mathbb{C}^{n}, w_{j}} p_{n, w_{j}} .
$$

Proof. Take $U$ as a union of balls with infinitesimal small radii about each $w_{j}$ (so that, for any $j=1, \ldots, m$, the germ $h_{w_{j}} \in \mathscr{O}_{\mathbb{C}^{n}, w_{j}}$ admits a representant $h_{j}$ in the connected component of $U$ that contains $w_{j}$ ). Take now the holomorphic function $f: U \rightarrow \mathbb{C}$ defined by $f_{\mid B_{j}}=h_{j}$ for $j=1, \ldots, m$ and then conclude appealing to Proposition 3.1. It also follows from Remark 3.2 that the system of coordinates $\left(\alpha_{0}\left(\left[\boldsymbol{h}_{\boldsymbol{w}}\right]\right), \ldots, \alpha_{N(\boldsymbol{p})-1}\left(\left[\boldsymbol{h}_{\boldsymbol{w}}\right]\right)\right)$ is that of the class of the polynomial (considered here in $\mathbb{C}[\boldsymbol{z}])$

$$
\begin{aligned}
& \operatorname{Lag}\left[h_{w_{1}}, \ldots, h_{w_{m}}\right] \\
& =\sum_{j=1}^{m} \sum_{\ell \in A_{w_{j}}} \frac{\partial^{|\ell|}}{\partial \boldsymbol{s}^{\ell}}\left[h_{w_{j}}\right]\left(w_{j}\right) \mathscr{Q}_{w_{j}, \ell}(\partial / \partial \boldsymbol{s})\left[\operatorname{det}\left[\boldsymbol{B}_{\boldsymbol{p}}(\boldsymbol{s}, \boldsymbol{z})\right]\right]\left(w_{j}\right) \\
& =\sum_{j=1}^{m} \sum_{\boldsymbol{\ell} \in A_{w_{j}}} \frac{\partial^{|\ell|}}{\partial \boldsymbol{s}^{\ell}}\left[\operatorname{det}\left[\boldsymbol{B}_{\boldsymbol{p}}(\boldsymbol{s}, \boldsymbol{z})\right]\right]\left(w_{j}\right) \mathscr{Q}_{w_{j}, \ell}(\partial / \partial \boldsymbol{s})\left[h_{w_{j}}\right]\left(w_{j}\right)
\end{aligned}
$$

in the basis $\mathscr{B} \underset{\mathbb{C}[\boldsymbol{z}] /(\boldsymbol{p}(\boldsymbol{z}))}{\prec \prec}$

\section{TWO NON-STANDARD INTERPOLATION PROBLEMS}

Let $\boldsymbol{p}=\left(p_{1}, \ldots, p_{n}\right)$ be a quasi-regular sequence in $\mathbb{C}[\boldsymbol{s}]$ and

$$
\mathscr{B}_{\mathbb{C}[\boldsymbol{s}] /(\boldsymbol{p})}^{\prec \prec}=\left\{\dot{\boldsymbol{s}}^{\boldsymbol{\beta}_{k}} ; k=0, \ldots, N(\boldsymbol{p})-1\right\}
$$

be a monomial basis of the $N(\boldsymbol{p})$-dimensional $\mathbb{C}$-vector space $\mathbb{C}[\boldsymbol{s}] /(\boldsymbol{p})$ which has previously been obtained thanks to the search for a Gröbner basis for $(\boldsymbol{p})$ with respect to the prescribed ordering $\prec \prec$ on monomials in $\mathbb{C}[s]$ (as recalled in section 2).

We will denote as in section 2 as $\boldsymbol{Q}_{\boldsymbol{p}}[\underset{\mathscr{B}}{\mathbb{C}[\boldsymbol{s}] /(\boldsymbol{p})}]$ (see $(2.7)$ ) the matrix of the quadratic non-degenerated form (2.6) which stands as the residual generator of the module $\operatorname{Hom}_{\mathbb{C}}(\mathbb{C}[\boldsymbol{s}] /(\boldsymbol{p}), \mathbb{C})$ (equipped with its structure of $\mathbb{C}[\boldsymbol{s}] /(\boldsymbol{p})$-module as described in section 2) constructed from the given quasi-regular sequence $\left(p_{1}, \ldots, p_{n}\right)$ of generators of the polynomial zero dimensional ideal $(\boldsymbol{p})$. The action of this generator is described as seen in section 2 by a standard list of Nœetherian operators

$$
\operatorname{Noeth}_{\boldsymbol{p}}[(\boldsymbol{p})]:=\left\{\mathscr{Q}_{w_{j}, \ell}(\partial / \partial \boldsymbol{s})_{\mid w_{j}} ; j=1, \ldots, m, \boldsymbol{\ell} \in A_{w_{j}}\right\}
$$

(see Definition 2.4).

We can now formulate (and indicate how to solve) the two following non-standard interpolation problems inspired by Problem 4.1 formulated in [3] in the univariate case.

Problem 4.1. Let $\boldsymbol{p}^{-1}(0)=\left\{w_{1}, \ldots, w_{m}\right\}$ and $1 \leq \mu \leq m$. Let $U$ be an open subset of $\mathbb{C}^{n}$ that contains $\left\{w_{1}, \ldots, w_{\mu}\right\}$. Let $a_{j, \ell}\left(j=1, \ldots, \mu, \boldsymbol{\ell} \in A_{w_{j}}\right)$, together with $c$, be 
$\left(\sum_{j=1}^{\mu} \# A_{w_{j}}\right)+1$ given complex numbers. Describe the $\mathbb{C}$-affine space of functions $f: U \rightarrow \mathbb{C}$ which are holomorphic in $U$ and moreover satisfy

$$
\sum_{j=1}^{\mu} \sum_{\ell \in A_{w_{j}}} a_{j, \ell} \mathscr{Q}_{w_{j}, \ell}[f]\left(w_{j}\right)=c .
$$

Problem 4.2. Let $w_{1}, \ldots, w_{\mu}$ be $\mu$ distinct points in $\mathbb{C}^{n}$, together with $\mu$ elements $\boldsymbol{\nu}_{1}, \ldots, \boldsymbol{\nu}_{\mu}$ in $\left(\mathbb{N}^{*}\right)^{n}$ (prescribed multi-vectors of multiplicities). Let $U$ be an open subset of $\mathbb{C}^{n}$ that contains $\left\{w_{1}, \ldots, w_{\mu}\right\}$ Let $a_{j, \ell}\left(j=1 \ldots, \mu, \boldsymbol{\ell} \in \mathbb{N}^{n}\right.$ such that $\left.\boldsymbol{\ell} \prec \boldsymbol{\nu}_{j}-\mathbf{1}\right)$, together with $c$, be $\left(\sum_{\ell=1}^{\mu} \prod_{j=1}^{n} \nu_{\ell, j}\right)+1$ given complex numbers. Describe the $\mathbb{C}$-affine space of functions $f: U \rightarrow \mathbb{C}$ which are holomorphic in $U$ and moreover satisfy

$$
\sum_{j=1}^{\mu} \sum_{\left\{\boldsymbol{\ell} \in \mathbb{N}^{n} ; \boldsymbol{\ell} \prec \boldsymbol{\nu}_{j}-\mathbf{1}\right\}} a_{j, \ell}\left(\frac{\partial^{|\ell|}}{\partial \boldsymbol{s}^{\ell}}\right)[f]\left(w_{j}\right)=c .
$$

We start by indicating how to solve Problem 4.1 in the particular case $\mu=m$. Recall that for each $j=1, \ldots, m$, for each $\ell \in A_{w_{j}}, \operatorname{Supp}\left(\mathscr{Q}_{w_{j}, \ell}\right) \subset A_{w_{j}}$, so that

$$
\mathscr{Q}_{w_{j}, \ell}(\partial / \partial \boldsymbol{s})=\sum_{\boldsymbol{\lambda} \in A_{w_{j}}} \tau_{j, \ell, \boldsymbol{\lambda}} \frac{\partial^{|\boldsymbol{\lambda}|}}{\partial \boldsymbol{s}^{\boldsymbol{\lambda}}}
$$

for some complex coefficients $\tau_{j, \ell, \lambda}$.

Lemma 4.3. Let $\left\{w_{1}, \ldots, w_{m}\right\}=\boldsymbol{p}^{-1}(0), U$ be an open subset of $\mathbb{C}^{n}$ containing $\boldsymbol{p}^{-1}(0)$ and the $a_{j, \ell}\left(j=1, \ldots, m, \ell \in A_{w_{j}}\right)$, together with $c$, be complex numbers. For each $j=1, \ldots, m$, let $h_{w_{j}}^{\boldsymbol{a}}$ be the germ in $\mathscr{O}_{\mathbb{C}^{n}, w_{j}}$ of $\boldsymbol{s} \longmapsto \sum_{\boldsymbol{\lambda} \in A_{j}} a_{j, \boldsymbol{\lambda}}\left(\boldsymbol{s}-w_{j}\right)^{\boldsymbol{\lambda}} / \boldsymbol{\lambda}$ ! and $\left[\boldsymbol{h}_{\boldsymbol{w}}^{\boldsymbol{a}}\right]=\left[h_{w_{1}}^{\boldsymbol{a}}, \ldots, h_{w_{m}}^{\boldsymbol{a}}\right]$. The following alternative holds :

- either the coordinate system $\left(\alpha_{0}\left(\left[\boldsymbol{h}_{\boldsymbol{w}}^{\boldsymbol{a}}\right]\right), \ldots, \alpha_{N(\boldsymbol{p})-1}\left(\left[\boldsymbol{h}_{\boldsymbol{w}}^{\boldsymbol{a}}\right]\right)\right)$ introduced in Proposition 3.3 is the null system, which amounts to say that

$$
\sum_{\boldsymbol{\lambda} \in A_{w_{j}}} \tau_{j, \ell, \boldsymbol{\lambda}} a_{j, \boldsymbol{\lambda}}=0 \quad \forall j=1, \ldots, m, \forall \boldsymbol{\ell} \in A_{w_{j}},
$$

in which case the set of holomorphic functions $f: U \rightarrow \mathbb{C}$ satisfying (4.2) is empty when $c \neq 0$ and is the whole space $H(U)$ when $c=0$;

- either the coordinate system $\left(\alpha_{0}\left(\left[\boldsymbol{h}_{\boldsymbol{w}}^{\boldsymbol{a}}\right]\right), \ldots, \alpha_{N(\boldsymbol{p})-1}\left(\left[\boldsymbol{h}_{\boldsymbol{w}}^{\boldsymbol{a}}\right]\right)\right)$ introduced in Proposition 3.3 is non-zero, in which case a function $f \in H(U)$ satisfies (4.2) if and only if

$$
\left[\alpha_{0}[f] \quad \ldots \quad \alpha_{N(\boldsymbol{p})-1)}[f]\right] \cdot \boldsymbol{Q}_{\boldsymbol{p}}[\underset{\mathscr{B}}{\mathbb{C}[\boldsymbol{s}] /(\boldsymbol{p})}]\left[\begin{array}{c}
\alpha_{0}\left(\left[\boldsymbol{h}_{\boldsymbol{w}}^{\boldsymbol{a}}\right]\right) \\
\vdots \\
\alpha_{N(\boldsymbol{p})-1}\left(\left[\boldsymbol{h}_{\boldsymbol{w}}^{\boldsymbol{a}}\right]\right)
\end{array}\right]=c,
$$

which means that the vector $\left(\alpha_{0}[f], \ldots, \alpha_{N(\boldsymbol{p})-1)}[f]\right)$ of the coordinates of $f$ in $H(U) /\left(\sum_{1}^{n} H(U) p_{j}\right)_{\text {loc }}$ lies in a specific affine hyperplane $\Pi^{a}$ of $\mathbb{C}^{N}$ since the quadratic form (2.6) is non-degenerated.

Proof. For each $j=1, \ldots, m$, let $\psi_{j} \in \mathscr{D}(U,[0,1])$ with support in an arbitrary small neighborhood of $w_{j}$ (which does not contain any other zero of $\boldsymbol{p}$ and is such that the germ $h_{w_{j}}^{a}$ admits a representant still denoted as $h_{w_{j}}^{a}$ in it) such that furthermore $\psi_{j} \equiv 1$ 
about $w_{j}$. It follows from (2.12) and (2.10) that one can rewrite the left-hand side of (4.2) as

$$
\begin{aligned}
& \sum_{j=1}^{m}\left\langle\left(\bigwedge_{j=1}^{n} \bar{\partial}\left(1 / p_{j}\right)\right)(\boldsymbol{s}), f(\boldsymbol{s}) h_{w_{j}}^{\boldsymbol{a}}(\boldsymbol{s}) \psi_{j}(\boldsymbol{s}) d s_{1} \wedge \cdots \wedge d s_{n}\right\rangle \\
&=\left\langle\left(\bigwedge_{j=1}^{n} \bar{\partial}\left(1 / p_{j}\right)\right)(\boldsymbol{s}), \operatorname{Lag}[f](\boldsymbol{s}) \operatorname{Lag}\left[\boldsymbol{h}_{\boldsymbol{w}}^{\boldsymbol{a}}\right](\boldsymbol{s})\left(\sum_{j=1}^{m} \psi_{j}(\boldsymbol{s})\right) d s_{1} \wedge \cdots \wedge d s_{n}\right\rangle \\
&=\operatorname{Res}\left[\begin{array}{c}
\operatorname{Lag}[f](\boldsymbol{s}) \operatorname{Lag}\left[\boldsymbol{h}_{\boldsymbol{w}}^{\boldsymbol{a}}\right](\boldsymbol{s}) d s_{1} \wedge \cdots \wedge d s_{n} \\
p_{1}(\boldsymbol{s}), \ldots, p_{n}(\boldsymbol{s})
\end{array}\right] \\
&=\left[\begin{array}{ll}
\alpha_{0}[f] & \ldots \\
\left.\alpha_{N(\boldsymbol{p})-1)}[f]\right] \cdot \boldsymbol{Q}_{\boldsymbol{p}}\left[\mathscr{B}_{\mathbb{C}[\boldsymbol{s}] /(\boldsymbol{p})}^{\prec \prec}\right] \\
\alpha_{0}\left(\left[\boldsymbol{h}_{\boldsymbol{w}}^{\boldsymbol{a}}\right]\right) \\
\vdots \\
\alpha_{N(\boldsymbol{p})-1}\left(\left[\boldsymbol{h}_{\boldsymbol{w}}^{\boldsymbol{a}}\right]\right)
\end{array}\right] .
\end{aligned}
$$

We now conclude using the fact that the quadratic form $\boldsymbol{Q}_{\boldsymbol{p}}$ is non degenerated. The first situation in the alternative corresponds precisely (thanks to the role of the Noetherian operators in the realisation of duality, see (2.14)) to the fact that the system of linear relations (4.5) holds.

We may now state the solution to Problem 4.1.

Theorem 4.4. Let $\boldsymbol{p}^{-1}(0)=\left\{w_{1}, \ldots, w_{m}\right\}$ and $1 \leq \mu \leq m$. Let $U$ be an open subset of $\mathbb{C}^{n}$ that contains $\left\{w_{1}, \ldots, w_{\mu}\right\}$. Let $a_{j, \ell}\left(j=1, \ldots, \mu, \boldsymbol{\ell} \in A_{w_{j}}\right)$, together with $c$, be $\left(\sum_{j=1}^{\mu} \# A_{w_{j}}\right)+1$ given complex numbers. For each $j=1, \ldots, \mu$, let $h_{w_{j}}^{\boldsymbol{a}}$ be the germ in $\mathscr{O}_{\mathbb{C}^{n}, w_{j}}$ of $\boldsymbol{s} \longmapsto \sum_{\boldsymbol{\lambda} \in A_{j}} a_{j, \boldsymbol{\lambda}}\left(\boldsymbol{s}-w_{j}\right)^{\boldsymbol{\lambda}} / \boldsymbol{\lambda}$ !. Let

$$
\left[\boldsymbol{h}_{\boldsymbol{w}}^{\boldsymbol{a}}\right]=\left[h_{w_{1}}^{\boldsymbol{a}}, \ldots, h_{w_{\mu}}^{\boldsymbol{a}}, 0_{w_{\mu+1}}, \ldots, 0_{w_{n}}\right] .
$$

The following alternative then holds :

- either the coordinate system $\left(\alpha_{0}\left(\left[\boldsymbol{h}_{\boldsymbol{w}}^{\boldsymbol{a}}\right]\right), \ldots, \alpha_{N(\boldsymbol{p})-1}\left(\left[\boldsymbol{h}_{\boldsymbol{w}}^{\boldsymbol{a}}\right]\right)\right)$ introduced in Proposition 3.3 is the null system, which amounts to say that

$$
\sum_{\boldsymbol{\lambda} \in A_{w_{j}}} \tau_{j, \ell, \boldsymbol{\lambda}} a_{j, \boldsymbol{\lambda}}=0 \quad \forall j=1, \ldots, \mu, \forall \boldsymbol{\ell} \in A_{w_{j}},
$$

in which case the set of holomorphic functions $f: U \rightarrow \mathbb{C}$ satisfying (4.2) is empty when $c \neq 0$ and is the whole space $H(U)$ when $c=0$;

- either the coordinate system $\left(\alpha_{0}\left(\left[\boldsymbol{h}_{\boldsymbol{w}}^{\boldsymbol{a}}\right]\right), \ldots, \alpha_{N(\boldsymbol{p})-1}\left(\left[\boldsymbol{h}_{\boldsymbol{w}}^{\boldsymbol{a}}\right]\right)\right)$ introduced in Proposition 3.3 is non-zero, in which case a function $f \in H(U)$ satisfies (4.2) if and only if (4.5) holds, which means that the vector $\left(\alpha_{0}[f], \ldots, \alpha_{N(\boldsymbol{p})-1)}[f]\right)$ of the coordinates of $f$ in $H(U) /\left(\sum_{1}^{n} H(U) p_{j}\right)_{\text {loc }}$ lies in a specific affine hyperplane $\Pi^{a}$ of $\mathbb{C}^{N}$ since the quadratic form (2.6) is non-degenerated.

Proof. One may assume that $\mu<n$ since the result is already proved when $\mu=m$ (Lemma 4.3).

Let us first assume that $\partial U$ does not contain any of the points $w_{j}$ such that $\mu<j \leq m$. Let $\widetilde{U}$ be the union of $U$ with open balls $B\left(w_{j}, \varepsilon_{j}\right), j=\mu+1, \ldots, n$, where $\varepsilon_{j}$ is strictly smaller than the distance from $w_{j}$ to $\bar{U}$. Any holomorphic function $f: U \rightarrow \mathbb{C}$ which satisfies (4.3) can be considered as $\widetilde{f}_{\mid \widetilde{U}}$ where $\widetilde{f}: \widetilde{U} \rightarrow \mathbb{C}$ satisfies (4.2) with $\tilde{a}_{j, \ell}=a_{j, \ell}$ for any $j=1, \ldots, \mu$ and any $\ell \in A_{w_{j}}, \tilde{a}_{j, \ell}=0$ for any index $j=\mu+1, \ldots, m$ and any $\ell \in A_{w_{j}}, \tilde{c}=c$. Conversely, given any such holomorphic solution $\tilde{f}: \tilde{U} \rightarrow \mathbb{C}$ of 
(4.2) in $\widetilde{U}$ (with the $a_{j, \ell}$ replaced by $\tilde{a}_{j, \ell}$ ), it restricts to $U$ as a solution of (4.3). The conclusion of Theorem 4.4 follows then from that of Lemma 4.3 ,

Consider now the case when $\partial U$ may contain some $w_{j}$ for $j=\mu+1, \ldots, m$. Let $\left(U_{k}\right)_{k \geq 0}$ be an increasing sequence of open subsets such that $\left\{w_{1}, \ldots, w_{\mu}\right\} \subset U_{k} \subset \overline{U_{k}} \subset U$ which exhausts $U$. It is equivalent to say that $f: U \rightarrow \mathbb{C}$ is solution of (4.2) in $U$ or that for any $k \in \mathbb{N}$, its restriction $f_{\mid U_{k}}$ is solution of (4.2) in $U_{k}$ (the data $a_{w_{j}, \ell}$ and $c$ remaining unchanged). For any $k \geq 0$, we showed that the alternative proposed in the statement of Theorem 4.4 hold. As pointed out in Remark 3.2. we also know that the coordinate system $\left(\alpha_{0}[f], \ldots, \alpha_{N(\boldsymbol{p})-1}[f]\right)$ ( $f$ being arbitrarily continued in $\left.\widetilde{U}_{k}=U_{k} \cup \bigcup_{j=\mu+1}^{m} B\left(w_{j}, d\left(w_{j}, \overline{U_{k}}\right) / 2\right)\right)$ does depend only of the germs of $f$ at the points $w_{j}$ for $j=1, \ldots, \mu$. On the other hand the condition on $\left[\boldsymbol{h}_{\boldsymbol{w}}^{\boldsymbol{a}}\right]$ which governs the alternative proposed in the statement of Theorem 4.4 (when $U=U_{k}$ ) does not depend on $k$. Hence this alternative still holds in the limit case $U_{\infty}=U$. Theorem 4.4 is thus proved in general.

Consider as an example the particular situation where $\boldsymbol{p}$ is a sequence of univariate monic polynomials respectively in the variables $s_{1}, \ldots, s_{n}$, as in the series of Examples 2.1 till 2.5. In this case, the $p_{j}$ being as in (2.8), the set $A_{w}$ which is related to the point $w=\left(\xi_{1, \kappa_{1}}, \ldots, \xi_{n, \kappa_{n}}\right) \in \boldsymbol{p}^{-1}(0)$ is

$$
A_{w}=\left\{\boldsymbol{\ell} \in \mathbb{N}^{n} ; \boldsymbol{\ell} \prec \boldsymbol{\nu}_{w}(\boldsymbol{p}):=\left(\nu_{1, \kappa_{1}}, \ldots, \nu_{n, \kappa_{n}}\right)-\mathbf{1}\right\},
$$

with cardinal $\prod_{\ell=1}^{n} \nu_{\ell, \kappa_{\ell}}$. Since one has in this case

$$
\sum_{j=1}^{m} \# A_{w_{j}}=N(\boldsymbol{p})=\operatorname{dim}_{\mathbb{C}}(\mathbb{C}[\boldsymbol{s}] /(\boldsymbol{p})),
$$

the first alternative in Lemma 4.3 leads in this particular case to $a_{w_{j}, \boldsymbol{\lambda}}=0$ for any $j=1, \ldots, m$, for any $\boldsymbol{\lambda} \in A_{w_{j}}$ for dimension reasons. This holds also for which what concerns the first alternative in Theorem 4.4 in the case $1 \leq \mu<m$ : it boils down in this case to the conditions $a_{j, \boldsymbol{\lambda}}=0$ for any $j=1, \ldots, \mu$, for any $\ell \in \mathbb{N}^{n}$ such that $\ell \prec \nu_{w}(p)-1$.

Consider now $\mu \geq 1$ distinct points in $\mathbb{C}^{n}$, paired with vectors of prescribed multiplicities $\boldsymbol{\nu}_{1}, \cdots, \boldsymbol{\nu}_{\mu}$ in $\left(\mathbb{N}^{*}\right)^{n}$ as in Problem 4.2. If $w_{j}=\left(\xi_{j, 1}, \ldots, \xi_{j, n}\right)$, let us form the $n$ univariate monic polynomials

$$
p_{j}\left(s_{j}\right):=\prod_{\ell=1}^{\mu}\left(s_{j}-\xi_{\ell, j}\right)^{\nu_{\ell, j}} \in \mathbb{C}\left[\boldsymbol{s}_{j}\right], \quad j=1, \ldots, n .
$$

Let $\boldsymbol{d}=\left(\operatorname{deg} p_{1}, \ldots, \operatorname{deg} p_{n}\right)$, where $\operatorname{deg} p_{j}=\sum_{\ell=1}^{\mu} \nu_{\ell, j}$ for $j=1, \ldots, n$. A monomial basis for $\mathbb{C}[s] /(\boldsymbol{p})$ is provided thanks to Euclid's algorithm in the separated variables $s_{1}, \ldots, s_{n}$ as

$$
\mathscr{B}_{\boldsymbol{p}}^{\text {euclid }}=\left\{\dot{\boldsymbol{s}}^{\boldsymbol{k}} ; \boldsymbol{k} \in \mathbb{N}^{n} \text { with } \boldsymbol{k} \prec \boldsymbol{d}-\mathbf{1}\right\} .
$$

From now on, one organizes this basis with respect to the lexicographical order on the multi-exposants of monomials in $\mathbb{C}[s]$. Keeping to such ordering, let

$$
\boldsymbol{Q}_{\boldsymbol{p}}^{\text {euclid }}:=\left[\operatorname{Res}\left[\begin{array}{c}
\boldsymbol{s}^{\boldsymbol{k}_{1}+\boldsymbol{k}_{2}} d s_{1} \wedge \cdots \wedge d s_{n} \\
p_{1}\left(s_{1}\right), \ldots, p_{n}\left(s_{n}\right)
\end{array}\right]\right]_{\substack{\boldsymbol{k}_{1}, \boldsymbol{k}_{2} \prec \boldsymbol{d}-\mathbf{1} \\
\boldsymbol{k}_{2} \in \mathbb{N}^{n}}} .
$$


The $\left(\boldsymbol{k}_{1}, \boldsymbol{k}_{2}\right)$ entry of such a matrix is obtained as the coefficient $\gamma_{\boldsymbol{k}_{1}, \boldsymbol{k}_{2}, \boldsymbol{d}-\mathbf{1}}$ of $\boldsymbol{s}^{\boldsymbol{d}-\mathbf{1}}$ in the expansion as a geometric series about $\mathbf{0}$ of

$$
\boldsymbol{s} \mapsto \boldsymbol{s}^{\boldsymbol{k}_{1}+\boldsymbol{k}_{2}} \prod_{j=1}^{n}\left(\frac{1}{1+\frac{p_{j}\left(s_{j}\right)-s_{j}^{d_{j}}}{s_{j}}}\right)=\sum_{\left\{\boldsymbol{\kappa} \in \mathbb{Z}^{n} ; \kappa \prec \boldsymbol{k}_{1}+\boldsymbol{k}_{2}\right\}} \gamma_{\boldsymbol{k}_{1}, \boldsymbol{k}_{2}, \boldsymbol{\kappa}} \boldsymbol{s}^{\boldsymbol{\kappa}} .
$$

For each $j=1, \ldots, \mu$, let $\psi_{j} \in \mathscr{D}\left(\mathbb{C}^{n},[0,1]\right)$ be a test-function which is identically equal to 1 near $w_{j}$ and identically equal to 0 about any point in $\boldsymbol{p}^{-1}(0) \backslash\left\{w_{j}\right\}$. Given a list $\boldsymbol{a}=\left\{a_{j, \ell} ; j=1, \ldots, \mu, \boldsymbol{\ell} \in \mathbb{N}^{n}\right.$ with $\left.\boldsymbol{\ell} \prec \boldsymbol{\nu}_{j}-\mathbf{1}\right\}$, consider the Lagrange interpolator $\operatorname{Lag}\left[\boldsymbol{h}_{\boldsymbol{w}}^{\boldsymbol{a}}\right] \in \mathbb{C}[\boldsymbol{z}]$ defined as

$$
\begin{aligned}
\operatorname{Lag}\left[\boldsymbol{h}_{\boldsymbol{w}}^{\boldsymbol{a}}\right](\boldsymbol{z})=\sum_{j=1}^{\mu}\left\langle\left(\bigwedge_{j=1}^{n} \bar{\partial}\left(1 / p_{j}\right)\right)(\boldsymbol{s}),\right. & \\
\left(\sum_{\boldsymbol{\ell} \prec \boldsymbol{\nu}_{j}-\mathbf{1}} a_{j, \ell} \frac{\left(\boldsymbol{s}-w_{j}\right)^{\ell}}{\boldsymbol{\ell} !}\right)\left(\prod_{j=1}^{n} \frac{p_{j}\left(z_{j}\right)-p_{j}\left(s_{j}\right)}{z_{j}-s_{j}}\right) & \left.\psi_{j}(\boldsymbol{s}) d s_{1} \wedge \cdots \wedge d s_{n}\right\rangle \\
& =\sum_{\left\{\boldsymbol{k} \in \mathbb{N}^{n} ; \boldsymbol{k} \prec \boldsymbol{d}-\mathbf{1}\right\}} \tau_{\boldsymbol{k}}(\boldsymbol{a}) \boldsymbol{z}^{\boldsymbol{k}} .
\end{aligned}
$$

One can now state the following result with respect to Problem 4.2 .

Theorem 4.5. Let $w_{1}, \ldots, w_{\mu}$ be $\mu$ distinct points in $\mathbb{C}^{n}$, together with $\mu$ elements $\boldsymbol{\nu}_{1}, \ldots, \boldsymbol{\nu}_{\mu}$ in $\left(\mathbb{N}^{*}\right)^{n}$. Let $U$ be an open subset of $\mathbb{C}^{n}$ that contains $\left\{w_{1}, \ldots, w_{\mu}\right\}$. Let $a_{j, \boldsymbol{\ell}}$ $\left(j=1 \ldots, \mu, \boldsymbol{\ell} \in \mathbb{N}^{n}\right.$ such that $\left.\boldsymbol{\ell} \prec \boldsymbol{\nu}_{j}-\mathbf{1}\right)$, together with $c$, be $\left(\sum_{\ell=1}^{\mu} \prod_{j=1}^{n} \nu_{\ell, j}\right)+1$ given complex numbers such that the $a_{j, \boldsymbol{\ell}}$ (for $j=1, \ldots, \mu, \boldsymbol{\ell} \prec \boldsymbol{\nu}_{j}$ ) are not all equal to 0 . An holomorphic function $f: U \rightarrow \mathbb{C}$ satisfies (4.3) if and only if there are coefficients $\alpha_{\boldsymbol{k}}, \boldsymbol{k} \prec \boldsymbol{d}$, where $d_{j}=\sum_{\ell=1}^{\mu} \nu_{\ell, j}$ for $j=1, \ldots, \mu$, such that

$$
f(s)=\sum_{\left\{\boldsymbol{k} \in \mathbb{N}^{n} ; \boldsymbol{k} \prec \boldsymbol{d}-\mathbf{1}\right\}} \alpha_{\boldsymbol{k}} \boldsymbol{s}^{\boldsymbol{k}}+g(\boldsymbol{s})
$$

where $g$ is an holomorphic function in $U$ which belongs locally to the ideal generated by the univariate polynomials $p_{j}\left(s_{j}\right)=\sum_{\ell=1}^{\mu}\left(s_{j}-w_{\ell, j}\right)^{\nu, j}(j=1, \ldots, n)$ and $\left(\alpha_{\boldsymbol{k}}\right)_{\boldsymbol{k} \prec \boldsymbol{d}-\mathbf{1}}$ satisfies

$$
\left[\begin{array}{lll}
\alpha_{\mathbf{0}} & \cdots & \alpha_{\boldsymbol{d}-\mathbf{1}}
\end{array}\right] \cdot \boldsymbol{Q}_{\boldsymbol{p}}^{\text {euclid }} \cdot\left[\begin{array}{c}
\tau_{\mathbf{0}}(\boldsymbol{a}) \\
\vdots \\
\tau_{\boldsymbol{d}-\mathbf{1}}(\boldsymbol{a})
\end{array}\right]=c
$$

where the coefficients $\tau_{\boldsymbol{k}}(\boldsymbol{a})\left(\boldsymbol{k} \in \mathbb{N}^{n}, \boldsymbol{k} \prec \boldsymbol{d}-\mathbf{1}\right)$ are those of the Lagrange interpolator (4.8).

Proof. This is an immediate application of Theorem 4.4 .

\section{Conclusions}

We have presented a new algebraic approach, based on residue theory and duality (see [9, 11, 12]) to solve a scalar interpolation problem in several complex variable. In a future work we plan to exploit the present methods in the matricial case to study the counterpart of the bitangential interpolation problem (see e.g. [6]) in the present setting. We focused here on the algebraic point of view. Hilbert space constraints will be considered elswhere. Both the methods and results are different from the ones 
classically related up to now to interpolation in the Drury-Arveson space or Schur multipliers and Schur-Agler classes (see [1, 4, 5, 7, for the latter).

\section{REFERENCES}

[1] J. Agler. On the representation of certain holomorphic functions defined on a polydisk, volume 48 of Operator Theory: Advances and Applications, pages 47-66. Birkhäuser Verlag, Basel, 1990.

[2] I.A. Aizenberg and A. P. Yuzhakov. Integral representations and residues in multidimensional complex analysis, volume 58 of Translations of Mathematical Monographs. American Mathematical Society, Providence, RI, 1983. Translated from the Russian by H. H. McFaden, Translation edited by Lev J. Leifman.

[3] D. Alpay, P. Jorgensen, I. Lewkowicz, and D. Volok. A new realization of rational functions, with applications to linear combination interpolation, the Cuntz relations and kernel decompositions. Complex Var. Elliptic Equ., 61(1):42-54, 2016.

[4] D. Alpay and H.T. Kaptanoğlu. Some finite-dimensional backward shift-invariant subspaces in the ball and a related interpolation problem. Integral Equation and Operator Theory, 42:1-21, 2002.

[5] J.A. Ball and V. Bolotnikov. Realization and interpolation for Schur-Agler-class functions on domains with matrix polynomial defining function in $C^{n}$. J. Funct. Anal., 213(1):45-87, 2004.

[6] J.A. Ball, I. Gohberg, and L. Rodman. Interpolation of rational matrix functions, volume 45 of Operator Theory: Advances and Applications. Birkhäuser Verlag, Basel, 1990.

[7] J.A. Ball and D.S. Kaliuzhnyi-Verbovetskyi. Schur-Agler and Herglotz-Agler classes of functions: positive-kernel decompositions and transfer-function realizations. Adv. Math., 280:121-187, 2015.

[8] C.A. Berenstein, R. Gay, A. Vidras, and A. Yger. Residue currents and Bezout identities, volume 114 of Progress in Mathematics. Birkhäuser Verlag, Basel, 1993.

[9] C.A. Berenstein and A. Yger. Residue calculus and effective Nullstellensatz. Amer. J. Math., 121(4):723-796, 1999.

[10] J.-Y. Boyer and M. Hickel. Extension dans un cadre algébrique d'une formule de Weil. Manuscripta Math., 98(2):195-223, 1999.

[11] J. P. Cardinal and B. Mourrain. Algebraic approach of residues and applications. In The mathematics of numerical analysis (Park City, UT, 1995), volume 32 of Lectures in Appl. Math., pages 189-210. Amer. Math. Soc., Providence, RI, 1996.

[12] M. Elkadi and B. Mourrain. Introduction à la résolution des systèmes polynomiaux, volume 59 of Mathématiques \& Applications (Berlin) [Mathematics \& Applications]. Springer, Berlin, 2007.

[13] A. Gleason. The Cauchy-Weil theorem. J. Math. Mech., 12:429-444, 1963.

[14] Z. Jelonek. On the effective Nullstellensatz. Invent. Math., 162(1):1-17, 2005.

[15] A. Płoski. On the Noether exponent. Bull. Soc. Sci. Lett. Lódz, 40(1-10):23-29 (1991), 1990.

[16] H. Samuelsson. Analytic continuation of residue currents. Ark. Mat., 47(1):127-141, 2009.

[17] A. Tsikh. Multidimensional residues and their applications, volume 103 of Translations of Mathematical Monographs. American Mathematical Society, Providence, RI, 1992. Translated from the 1988 Russian original by E. J. F. Primrose.

[18] A. Tsikh and A. Yger. Residue currents. J. Math. Sci. (N. Y.), 120(6):1916-1971, 2004. Complex analysis.

[19] A. Weil. L'intégration dans les groupes topologiques et ses applications. Actual. Sci. Ind., no. 869. Hermann et Cie., Paris, 1940. [This book has been republished by the author at Princeton, N. J., 1941.].

Schmid College of Science and Technology, Chapman University, One University Drive Orange, California 92866, USA

E-mail address: alpay@chapman.edu

$U R L:$ http://www1.chapman.edu/ alpay

Institut de Mathématiques, Université de Bordeaux. 351 Cours de la Libération, 33405 Talence, France

E-mail address: Alain.Yger@math.u-bordeaux.fr

$U R L:$ http://www.math.u-bordeaux.fr/ ayger 